\title{
Reflexiones sobre el papel de trabajadores de arte feministas.
}

\author{
Reflections about the role of feminist art \\ workers.
}

\section{Reflexões sobre o papel das trabalhadoras de arte feministas.}

\author{
ELENA BANGUESES ${ }^{1}$ \\ THOMAS APOSTOLOU²
}

\section{Resumen:}

Reflexionamos sobre el estado actual del feminismo en las artes y las profesiones que las rodean. A partir de las diversas experiencias profesionales de los autores en el campo del arte contemporáneo, en museos, galerías, universidades y otras instituciones, comparamos las fuentes bibliográficas con la realidad a la que se enfrenta el feminismo en el mundo del arte. Identificamos los problemas que plagan las experiencias de las profesionales del arte a gran y pequeña escala y reflexionamos sobre las formas de reaccionar ante ellos y repensarlos, concluyendo con algunas sugerencias prácticas sobre cómo cuestionar las restricciones existentes y promover los feminismos en el mundo del arte.

Palabras clave: Feminismo, mundo del arte, profesionales del arte.

\footnotetext{
1 Historiadora del arte por la Universidad de Santiago de Compostela. Master en Gestión Cultural por la City University of London, Reino Unido. Gestora cultural y comisaria independiente - elenabangueses@ gmail.com - https://orcid.org/0000-0002-6857-7637

2 Facultad de Bellas Artes, Universidad de Vigo, Pontevedra, España. Doctorado en Creación e Investigación en Arte Contemporáneo. https://orcid.org/0000-0001-6447-0552
} 


\section{Abstract:}

We reflect on the present state of feminism in the arts and the professions surrounding them. Drawing from the authors diverse professional experiences in contemporary arts, in museums, galleries, universities and other institutions, we compare bibliographic sources with the reality feminism faces in the art world. We identify problems that plague artworkers' experiences on large and small scale and reflect on ways to react to them and rethink them concluding with some practical suggestions on how to bring existing strictures into question and promote feminisms in the art world.

Key words: Feminism, art world, art workers.

\section{Resumo}

Neste artigo apresentamos uma reflexão sobre o estado atual do feminismo nas artes e nas profissões que as cercam. Com base nas diversas experiências profissionais de autoras da área da arte contemporânea, seja em museus, galerias, universidades e outras instituições, comparamos as fontes bibliográficas com a realidade que o feminismo enfrenta no mundo da arte. Identificamos os problemas que assolam as experiências dos grandes e pequenos profissionais da arte e refletimos sobre as formas de reagir e repensá-los, concluindo com algumas sugestões práticas sobre como desafiar as restrições existentes e promover o feminismo no mundo da arte.

Palavras-chave: Feminismo, mundo da arte, profissionais da arte.

\section{Introducción}

La realidad actual en el mundo del arte sigue estando dominada por ciertos grupos y el interés hacia el término feminismo ha aumentado. El feminismo de hoy, con toda su diversidad y su historia, lleva más de una década cogiendo inercia, y recientemente ha llegado a formar parte de la cultura y el discurso "mainstream". La idea central de este artículo proviene de la comunicación Reflexiones sobre mujer y comisariado, que formó parte de las Jornadas Resiliencia, mujer y arte en 2021 importada por una de las autoras de este artículo, Elena Bangueses. Esta adaptación de la idea inicial, trata de combinar las experiencias de los dos autores desde distintos puntos de vista, como profesionales en el mundo del arte con compromiso feminista. A través de comparación bibliográfica, junto a las experiencias vividas, proponemos unas recomendaciones sobre cómo acercar la 
actualidad del arte contemporáneo y el comisariado a un futuro realmente inclusivo y representativo.

Hoy en día, tiene poco sentido hablar del feminismo en singular, como si fuera tan estructurado como en la segunda ola del feminismo. La ideología y la teoría, han llegado a tanta pluralidad, que si queremos ser honestos deberíamos hablar de feminismos. Este, quizás sea el efecto más radical que tuvo este empuje reciente que nos ha llevado a la situación actual. Esta pluralidad, que hace unas décadas, podría haber significado dispersión en los rangos, hoy es inevitable y un recurso imprescindible. Esta expansión de lo que se puede considerar feminismo, por lo menos en el ámbito del arte y el comisariado, no significa que el término se pueda aplicar de manera arbitraria, existen definiciones rigurosas y a la vez expansivas (WINANT, 2016, p. 138-143). Feminismo, en el contexto de este texto, se refiere a esta pluralidad expansiva de todos los feminismos de la actualidad, así como, pueden servir, unidos por sus diferencias, para discutir una variedad de temas a través del arte (REILLY, 2010, p. 156-173).

\section{El Mundo del Arte}

El mundo del arte no es inmune a lo que afecta a todos los demás aspectos de la vida. Lo forman un conjunto de personas e instituciones, la mayoría de las cuales no promueven la igualdad. Las cifras en el mercado del arte y en las instituciones públicas son claras, cualquier artista y comisaria, que no sea hombre blanco, está mal representada y devaluada.

Desde 1970, ha surgido una significativa investigación feminista enfocada en la baja representación de las mujeres, y otros grupos, en instituciones de arte públicas y privadas, pero la situación a nivel estadístico no ha cambiado tanto (BROWN, 2020). En teoría, la situación sociopolítica generada por la crisis financiera de la primera década de este milenio, está relacionada con el resurgimiento feminista (HORNE; TOBIN, 2014, p. 75-83), pero las cifras no cuentan lo mismo. Lamentablemente, las mujeres y demás personas que no tienen cabida en las perspectivas binarias de género y los estereotipos sexistas, raciales y clasistas promovidos por el machismo institucional, siguen siendo menos visibles y valoradas. Por ejemplo, el Observatorio de la asociación de Mujeres en las Artes Visuales (MAV) informa que en 2020 las cifras siguen siendo, con dos excepciones, desiguales en la mayoría de las ferias de arte de Madrid (MAV, 2020). Los datos del I Informe sobre la aplicación de la Ley de Igualdad en el ámbito de la cultura dentro del marco competencial del Ministerio de Cultura y Deporte de España tampoco pinta bien, aunque según los datos recogidos hasta 2018, un mayor número de mujeres 
crea, así como, participa en otras funciones en las artes plásticas. Museos, como el Prado, siguen comprando desproporcionalmente más obras de hombres, programan más exposiciones comisariadas por hombres y publican libros con editores y colaboradores mayoritariamente masculinos (ANLLO VENTO, 2020). Las cifras tampoco cuentan otras historias fuera del contexto de España (LOZANO, 2019).

¿Qué significa esto para una comisaria o una profesional en las artes visuales? Desde la experiencia podemos decir que el techo de cristal es real, y algunas nos damos cuenta de él progresivamente y otras chocamos con él a toda velocidad. Podemos llegar a ser apreciadas y respetadas por nuestro trabajo, pero, para asumir una responsabilidad real, una mujer tiene que ser excepcional, incluso en los entornos que deberían ser más progresistas, aunque son la excepción. Eso no significa que una vez atravesado ese techo de cristal los problemas terminen.

El sexismo, el racismo y los estereotipos binarios de género son una batalla diaria, tanto a nivel personal como institucional. Reflexionemos sobre el siguiente escenario: La directora y la comisaria de un museo de arte contemporáneo, de los más reconocidos del mundo, debaten decisiones sobre el comisariado de la exposición de una de las artistas más vendidas del mundo, y al entrar el coleccionista que ha prestado las obras, las dos expertas toman las decisiones atendiendo a las opiniones y deseos de este. Esta situación, que por desgracia no es imaginaria, indica que el poder económico, político y social del patriarcado tampoco es imaginario, y se multiplica en ámbitos plagados por la precariedad, como la cultura.

Por lo tanto, desde el principio, debemos entender que estamos trabajando en un sistema construido por y para hombres. El sistema de arte sigue siendo clasista, promete una movilidad ascendente, pero esta la consiguen mayoritariamente hombres (DIMITRAKAKI, 2019, p. 513-531). Se están dando pasos, pero hasta ahora, los avances son solo como pequeñas grietas en el monolito del sistema, que apenas afectan a su superficie. Es descorazonador recordar el futuro prometedor de la primera década de los 2000, cuando pequeños cambios nos hicieron imaginar un futuro, donde el feminismo formaría uno de los ejes del comisariado de arte contemporáneo (FLETCHER, 2008, p. 108-113). Este futuro, por desgracia, nunca llegó, en su lugar surgieron otras corrientes, como el contramovimiento de la misoginia popular o las agendas de extrema derecha, que generalizando y polarizando, intentan quitar crédito al discurso feminista (PUENTE; ROMERO, 2020, p. 125-142).

La visibilidad tiene que ser uno de los objetivos, aunque no el único, puesto que pone el punto de mira en el problema y demuestra un potencial de cambio. Independientemente, una comisaria o gestora feminista, siempre debe trabajar para profundizar en estas primeras grietas prometedoras de los años pasados sobre el sistema establecido, a través de cada acto, cada exposición, cada red, cada 
intervención pública y privada. Tanto las pequeñas como las grandes ocasiones aportan.

Por otro lado, la visibilidad, o mejor dicho su conversión desde una herramienta a una meta, corre el peligro de convertirse en una artimaña. El consumismo, en ocasiones, ha convertido la visibilidad en producto, reduciendo su potencial de cambio político y social. Hablando estrictamente de comisariar arte contemporáneo, las mujeres comisarias, gerentes o artistas, en ocasiones se convierten en fachadas detrás de las cuales se esconde el machismo institucional. Políticos y medios de comunicación, nunca olvidan mencionar el género de una mujer cuando hablan de su trabajo. Nuestra experiencia profesional sobre estas actitudes esta perfectamente ilustrada por Maite Garbayo-Maeztu (2020), que reflexiona sobre la insistencia de los medios en hacer referencia a la exposición Yo, la peor de todas como una "exposición de mujeres artistas" o "de género". La comisaria especula, que esto sucede porque el establecimiento social encuentra dificultades para aceptar una exposición, con un gran número de artistas mujeres, como "normal". Habiendo compartido experiencias similares, estamos de acuerdo con la comisaria en que deberíamos evaluar nuestras estrategias, para evitar estas reacciones y falsas contextualizaciones de proyectos. Además, debemos mencionar que estos falsos caracterismos podrían también indicar una segregación institucional de "arte normal" y "arte de mujeres" dentro de una táctica que intenta ilegitimar y hacer difusas las demandas de los discursos feministas. Entonces, parece ser que las cifras que todas empujamos al igualar, tintan femenino un proyecto una vez que los hombres pasan a ser minoría. De hecho, a veces, el simple empleo del femenino en los títulos de proyectos, produce el mismo resultado. De todas maneras, si queremos trabajar hacia una multiplicidad y alejarnos de la tergiversación, al menos por ahora, tratar de equilibrar las cifras es una ruta viable.

Todo esto nos lleva a repensar cómo podría actuar una comisaria comprometida con el feminismo. Intentaremos evitar las definiciones, ya que, si algo hay que cultivar como feminista, es la incertidumbre y desconfianza en las estructuras rígidas. Una buena práctica es mantener una actitud de agudeza política y derivada de ideología y filosofía de género, a la vez que abrirse a nuevos modos y prácticas artísticas que quizás han quedado fuera del entorno feminista (JONES, 2016), es decir, ser conscientes del pasado y presente, pero con esperanza de cambio. Tenemos que ser conscientes que los 1970, para las artistas jóvenes pertenecen a la historia, y muchas de ellas han asimilado esta historia y sus lecciones en sus propios feminismos, sin necesariamente formar parte de ninguna estructura feminista. Nuestro discurso como trabajadoras de arte tiene que ser de duda, cultivando, discutiendo y dirigiendo incertidumbres. El feminismo debería ser un 
fenómeno cambiante, que se cuestiona a si mismo y desafía los modos de poder constantemente (WINANT, 2016, p. 138-143).

Al mismo tiempo, no podemos olvidar que el comisariado y la gestión cultural son trabajos pragmáticos. Ser pragmática y hábil es una virtud necesaria para una comisaria o gestora, incluso si el objetivo es producir cambios. La realidad de hoy en día se parece mucho a la de un mago, estamos creando valor cultural en un tiempo récord, con recursos muy limitados y debemos cumplir con grandes expectativas, es como sacar un conejo de un sombrero. Viendo las grandes exposiciones producidas durante las dos primeras décadas de los 2000, sobre feminismo o sobre artistas mujeres (dos cosas diferentes, pero que cuentan para las instituciones hacia la cuota de igualdad), nos puede parecer, que para contribuir, tenemos que llegar a ese nivel o hacer algo radical o nuevo. Todos tenemos nuestras ideologías y todas varían, pero es importante no perder nunca de vista los cambios que queremos ver en nuestra cultura y utilizar cada proyecto como un paso hacia esos cambios. Con esto, no queremos decir que los saltos no sean aconsejables, al contrario, admiramos a quienes los dan, a lo que nos referimos es que, si en cada proyecto damos un pequeño paso hacia el cambio que queremos ver en la cultura contemporánea, antes de que nos demos cuenta, tendremos el impulso necesario para dar un salto.

Deberíamos haber llegado ya a la época en que no hace falta alcanzar las vallas del patriarcado, sino tratar nuestra presencia y nuestros paradigmas como estándar. Mientras escribimos estas líneas, se publica la noticia de que Laurence des Cars va a ser la primera directora mujer del Museo de Louvre. Nos preguntamos, si las noticias del nombramiento del director actual en 2013, habían anunciado también que era un hombre en la primera línea. Parece ser, que ser mujer es una irregularidad, por esto una comisaria todavía tiene que asumir el papel de infiltrada. La crítica institucional es una herramienta eficaz para generar cambios y desafiar estructuras patriarcales, especialmente cuando viene desde dentro, aunque tiene sus limitaciones. Audre Lorde, cuando decía que las herramientas del maestro nunca desmantelarán su propia casa ${ }^{3}$, avisaba que el feminismo académico de su época, en los Estados Unidos, fuese incapaz de producir cambios si seguía ignorando temas como la homofobia, el racismo y la ilegalidad económica (LORDE, 2007, p. 110-114). Pero el discurso, de donde viene este texto, Lorde lo dio a una mayoría de mujeres blancas, ella misma se había infiltrado, ya que era la única ponente lesbiana y negra. Siendo consiente de la rareza de esta oportunidad, lanzó su crítica contra una academia que ignoraba gran parte del tema que estaban estudiando. ¿Qué debería hacer una feminista al conseguir un cargo

3 Traducción de la autora del titulo original de la charla de Audre Lorde en ingles: The Master's Tools Will Never Dismantle the Master's House 
institucional? Dejar la puerta abierta al entrar no es suficiente, debería también empezar a reconstruir la casa entera. Mejor expresado por Stella Rollig (2016) "La mala noticia es: no hay instituciones feministas. Y la buena noticia: pero hay feministas en las instituciones"4.

\section{Conclusiones}

En conclusión, se espera que una mujer, sea cual sea su campo, haga un esfuerzo extra, por lo que vale la pena dedicar este esfuerzo hacia el cambio que queremos ver en las artes y la cultura. Deberíamos trabajar por una cultura que represente y atienda a todos sus miembros por igual, inclusiva, alejada de los estereotipos de género binarios, pero también del racismo y la discriminación por edad. Esto requiere un esfuerzo extra para lograr una representación más justa y dar visibilidad a los colectivos ahora subrepresentados. Es vital estar al tanto del pasado, presente y futuro de nuestras profesiones, pero también de la historia feminista ligada a ellas. Debemos seguir apoyándonos unas en otras e impulsar nuestra visión de futuro en cada oportunidad. El cuestionamiento incesante, de las instituciones, de nosotras mismas, de los modos de producción artística y del rol curatorial, es fundamental. El mundo del arte está orientado a mantener narrativas y prácticas que refuerzan las jerarquías existentes, y todo este sistema se basa, en gran medida, en certezas y corroboraciones. Hacer preguntas y cultivar incertidumbres es una forma de socavar esta estructura y al mismo tiempo promover la pluralidad.

\section{Referencias}

ANLLO VENTO, Fátima. I Informe sobre la aplicación de la Ley de Igualdad en el ámbito de la cultura dentro del marco competencial del Ministerio de Cultura y Deporte: Observatorio de Igualdad de Género en el ámbito de la Cultura. [s.l.] : Ministerio de Cultura y Deporte de España, 2020. Disponível em: https:// www.culturaydeporte.gob.es/dam/jcr:067e3922-191c-4687-b880-39d683a4d8cf/ informe-de-igualdad.pdf. Acesso em: 10 abr. 2021.

BROWN, Sally. Feminist Curating: What it means and why it matters. Faculty \& Staff Scholarship. [S. l.], Faculty \& Staff Scholarship., 2020. Disponível em: https:// researchrepository.wvu.edu/faculty_publications/2947.

4 Traducción de los autores de original en inglés: The bad news is: there is no feminist institution. And the good news: but there are feminists in the institutions. 
DIMITRAKAKI, Angela. Masculinity, Art, and Value Extraction. Hoboken, NJ, USA: John Wiley \& Sons, Inc, 2019. p. 513-531 DOI: 10.1002/9781118929179.ch29. Disponível em: https://onlinelibrary.wiley.com/doi/abs/10.1002/9781118929179. ch29.

FLETCHER, Annie. On Feminism (Through a Survey of Several Recent Exhibitions). Afterall: A Journal of Art, Context and Enquiry. [S. l.], n. 17, Afterall: A Journal of Art, Context and Enquiry, p. 108-113, 2008. Disponível em: http:// www.jstor.org/stable/20711683.

GARBAYO-MAEZTU, Maite. Tergiversar, citar, tropezar: el comisariado como práctica feminista $=$ Shifting, citing, stumbling: curating as a feminist practice. Espacio, tiempo y forma. revista de la Facultad de Geografía e Historia / Serie 7, Historia del arte. [S. l.], n. 8, Espacio, tiempo y forma. revista de la Facultad de Geografía e Historia / Serie 7, Historia del arte, p. 47-74, 2020. DOI: 10.5944/ etfvii.8.2020.27478. Disponível em: https://doaj.org/article/d9b5b37729214c34be7fa9d591c39d4a.

HORNE, Victoria; TOBIN, Amy. an unfinished revolution in art historiography, or how to write a feminist art history. Feminist Review. [S. l.], n. 107, Feminist Review, p. 75-83, 2014. Disponível em: http://www.jstor.org/stable/24571890.

ISKIN, Ruth E. Feminism, Exhibitions and Museums in Los Angeles, Then and Now. Woman's Art Journal 37. [S. l.], v. 37, n. 1, Woman's Art Journal, p. 12-20, 2016. Disponível em: http://www.jstor.org/stable/26452050.

JONES, Amelia. Feminist Subjects versus Feminist Effects: The Curating of Feminist Art (or is it the Feminist Curating of Art?). 2016. Disponível em: https:// www.on-curating.org. Acesso em: 2020.

LORDE, Audre. The Master's Tools Will Never Dismantle the Master's House. Berkeley: Crossing Press, 2007. p. 110-114

LOZANO, Beatriz. Visualizing the Numbers: See Infographics Tracing the Representation of Women Artists in Museums and the Market. 2019. Disponível em: https://news.artnet.com/womens-place-in-the-art-world/visualizing-the-numbers-see-infographics-1654084. Acesso em: 2020.

MAV. FERIAS / LOS DATOS BAJO EL PRISMA DEL GÉNERO. AVANCE DEL INFORME MAV \#20. 2020. Disponível em: https://mav.org.es/ferias-arte-2020-mav/. Acesso em: 2021.

PUENTE, Sonia Núñez; ROMERO, Diana Fernández. La misoginia popular como 
contramovimiento: estudio de la resemiotización y los discursos manipulativos como desafíos contra el feminismo. Ex aequo (Oeiras, Portugal). [S. l.], n. 41, Ex aequo (Oeiras, Portugal), p. 125-142, 2020. DOI: 10.22355/exaequo.2020.41.08. Disponível em: http://www.scielo.mec.pt

REILLY, Maura. Curating Transnational Feminisms. Feminist Studies 36. [S. l.], v. 36, n. 1, Feminist Studies, p. 156-173, 2010. Disponível em: http://www.jstor.org/ stable/40608006.

ROBINSON, Hilary. Feminism meets the big exhibition: museum survey shows since 2005. On-curating. [S. l.], n. 29, On-curating, p. 29-39, 2016. Disponível em: https://www.on-curating.org/issue-29.html\#.YK5ot6gzabg.

ROLLIG, Stella. In search of the Feminist (in the) Institution. [s.l.] : CreateSpace Independent Publishing Platform, 2016. Disponível em: https://www.on-curating.org/issue-29-reader/in-search-of-the-feminist-in-the-institution.html\#. YK95y6gzabg.

WINANT, Carmen. Our Bodies, Online. Aperture. [S. l.], n. 225, Aperture, p. 138-143, 2016. Disponível em: http://www.jstor.org/stable/44404723. 